\title{
SYSTEMIC LUPUS ERYTHEMATOSUS AND DEMYELINIZING DISEASE: A CASE REPORT
}

\author{
Maria Marina da Nóbrega Carvalho', ${ }^{1,}$ Ana Beatriz Nogueira da Cruz ${ }^{1}$, Teresa Patricia Acebey Crespo ${ }^{1}$, Pablo Duarte Lima ${ }^{1}$ \\ 1.Centro Universitário de João Pessoa, João Pessoa (PB), Brazil. \\ *Corresponding author: marinanobrega96@hotmail.com
}

\section{BACKGROUND}

Multiple sclerosis (MS) and systemic lupus erythematosus (SLE) are autoimmune disorders. Multiple sclerosis courses with inflammation and chronic demyelination. Optic neuritis, optic myelitis, motor disorder, pain, spasticity, cognitive decline, fatigue, dysphagia, sphincter and sexual disorder, and paroxysmal symptoms may occur. Systemic lupus erythematosus is a multisystem disease with autoantibody production. It has a broad picture with malar eruption, discoid eruption, photosensitivity, oral ulcers, arthritis, serositis, kidney disease, neurological disorders, hematological disorders, positive ANA, fever, fatigue. Demyelinating syndrome, headache, movement disorder, myelopathy, seizures, acute confusional state, cognitive dysfunction, mononeuropathy, and polyneuropathy may occur. Brain MRI may have focal hyperintense lesions in the periventricular and subcortical white matter on T2 and may be identical to MS lesions.

\section{CASE REPORT}

A 29-year-old male patient with onset of 2018. He went to the hospital reporting severe, sudden, intermittent holocranial headache, in flares, without irradiation and without associated symptoms. He was diagnosed with migraine. Due to the persistence of the condition, he sought a neurologist who requested an MRI. Multiple sclerosis was suspected. Later, he reported dizziness, diplopia, severe weakness in the left side and mild in the right, muscle spasms, memory loss, visual problems, with scotomas and difficulty in focusing, imbalance associated with falls, mood changes, worsening of symptoms in extreme temperatures. She was diagnosed with MS and started natalizumab IV. In routine MRI, a lesion raised the hypothesis of rheumatologic disease. Blood count, ANA, RF, ESR, PCR, C3, C4 CH50, anti-DNA, urine summary, protein/creatinine were requested and referred to a rheumatologist. He presented a clinical picture with cutaneous lesions in the lower limbs and scalp, alopecia, arthralgia. Normal complement, ANA with homogeneous nuclear pattern 1:640, elevated CRP and ESR, negative FR. He was diagnosed with SLE and started hydroxychloroquine. Currently, there is numbness in the left third finger, constipation, nocturnal choking and lack of attention while driving. Follows up in outpatient follow-up.

\section{CONCLUSION}

Despite being a difficult disease to diagnose, when combined with clinical, laboratory and imaging findings, it was possible to identify MS associated with a typical picture of SLE. The coexistence of both in the same patient is quite rare. The differential diagnosis in the case described is challenging, especially when MS symptoms are associated with neurological involvement in SLE, making it difficult to distinguish which disease is prevailing. These are diseases of great significance and require multidisciplinary intervention, especially in neurology and rheumatology.

\section{KEYWORDS}

Systemic lupus erythematosus, Demyelinating disease, Rare, Differential diagnosis. 